\title{
Scale Invariance in Lateral Head Scans During Spatial Exploration
}

\author{
Chetan K. Yadav and Yoganarasimha Doreswamy* \\ National Brain Research Centre, NH-8, Manesar, Haryana 122051, India
}

(Received 28 April 2016; published 13 April 2017)

\begin{abstract}
Universality connects various natural phenomena through physical principles governing their dynamics, and has provided broadly accepted answers to many complex questions, including information processing in neuronal systems. However, its significance in behavioral systems is still elusive. Lateral head scanning (LHS) behavior in rodents might contribute to spatial navigation by actively managing (optimizing) the available sensory information. Our findings of scale invariant distributions in LHS lifetimes, interevent intervals and event magnitudes, provide evidence for the first time that the optimization takes place at a critical point in LHS dynamics. We propose that the LHS behavior is responsible for preprocessing of the spatial information content, critical for subsequent foolproof encoding by the respective downstream neural networks.
\end{abstract}

DOI: 10.1103/PhysRevLett.118.158104

A number of natural systems of diverse origin exhibit featured dynamics and share similar properties. Spatiotemporal scale invariance indicating fractality is one of them, and various power laws are usual formulations to illustrate such fractals. $P(X) \sim X^{-\alpha}$ is a form of power law expression, where probability of a variable $X$ decays following a power function with an exponent $\alpha$. Such fractals exhibiting power law distributions are ubiquitous [1]. Bak and colleagues [2,3] laid a conceptual foundation for a theory of "self-organized criticality (SOC)," which is adopted with modifications in various physical and natural systems [4], including biological [5] and neuronal [6] systems, unified by scale free features in their dynamics. Absence of a characteristic scale shows that the system is evolving continuously, and this spontaneous process benefits the system in many ways by posing its dynamics in a complex state of transition between order and complete randomness. This state is actually a critical state where the system is neither ordered nor random. Dynamics of many selforganized systems has been attributed to the presence of a "critical point" that has been shown to facilitate information processing, and has been proven in neuronal systems [6-8]. Even though the scale invariance has been reported in various spontaneous behaviors with a wider range of scaling exponents as compared to physical systems [9], its significance in sensory information management at behavioral level has not been reported. Since behavior is an act of response towards the environment, certain behaviors such as spatial

Published by the American Physical Society under the terms of the Creative Commons Attribution 3.0 License. Further distribution of this work must maintain attribution to the author(s) and the published article's title, journal citation, and DOI. navigation may require active management of the available sensory information during exploration.

Animals navigate in various environments ranging from fully familiar to completely novel situations and encounter an enormous amount of information on the positional and directional architecture of the environment (see [10] for a review). This information content is not only acquired but processed continuously during spatial exploration for memory formation and drafting a cognitive map of the given space [11]. Such information encoded in the form of spatial map is utilized upon recall during decision making, for example, rats adopting straight paths for rapid returns to home base location [12-14]. Animals have been reported to inherit an internal sense of direction [15], the neural correlates of which are the head direction cells [16] acting like an internal compass, reported to be used for homing through path integration mechanisms [17]. Further, it has been shown that both the idiothetic cues and external landmarks are capable of controlling activities of place cells as well as head direction cells [18]. To navigate successfully in space, rats not only need to keep track of their position and orientation within reference frame, but also need to update the same in order to minimize errors accumulating with time [19]. This requirement is achieved through "lateral head scanning" [20,21] behavior during pauses in their movement, wherein the animals move their head to access information about the external environment and calibrate their internal sense of direction. This event of the head's deviation from its original direction and return back to the same is known as a lateral head scan (LHS). LHS are performed during temporally structured nonrandom pauses [22-24], and are considered to be attentive and investigatory in nature [25,26]. Recently, Monaco et al. [27] have shown potentiation of place fields and increased theta power at LHS sites, establishing a connection between LHS and the neural correlates of 
spatial navigation. The role of LHS behavior during spatial information processing might not be limited only to conveying relevant sensory information to the neural correlates of navigation, but may be critical for optimization of the same beforehand. This is because, with a limited number of scans performed by the animal during exploration, information management may be achieved only by sharing the sensory load (experiences) through numerous interactions in a complex manner. The present study tests the hypothesis that LHS dynamics is a self-organized state of complexity maintaining itself at a critical point. This critical point not only expands the system's capabilities to handle enormous sensory load, but also facilitates processing of the spatial information. We report that the LHS dynamics contributing to spatial navigation is a scale free phenomenon, illustrated through power law distributions of LHS probabilities for their magnitudes, durations, and interevent intervals. A SOC state of LHS dynamics is being proposed. We substantiate our observations of LHS behavior to be an example of criticality by showing deviations in power law exponents under the influence of partial anesthesia (PA) and amphetamine (AMP), where the normal spatial exploratory process is altered.

Sixteen Long Evans rats (3-4 months old weighing between 400 and 500 grams) were allowed individually to freely explore on a completely novel elevated platform for 90 minutes during the dark phase of their circadian cycle. The experimental room was illuminated with a ceiling mounted light source and surrounded with a black circular curtain having four salient distal landmarks hanging on it. Rats performed LHS during intermittent pauses structured within the animal's movement profile. LHS events were detected by processing the video data of the animal behavior and time series of these events were analyzed offline (see Supplemental Material [28] for a description of experimental subjects and methods used for acquiring and analyzing the data).

First, the behavioral experimental observations in rats under normal conditions (NC) $(n=8)$ is presented, followed by comparison of experimental observations in rats under the influence of PA $(n=4)$ and AMP $(n=4)$. Figure 1(a) shows a representative LHS event during the pause in movement in the NC rat. An exemplary rat was moving before making a scan, and approached the "LHS site" as indicated by $5.03 \mathrm{~cm}$ displacement of its centroid $(+)$ from $4 \mathrm{sec}$ before scan start to initiation of the same. At this site, the rat performed a scan of $3.2 \mathrm{sec}$ duration during which the centroid position was almost constant. LHS consisted of upstream, transition, and downstream segments. During the upstream phase, nose position and direction (functions of head turning) deviated from their instantaneous values preceding the scan's start, fluctuated or remained constant during transition, and approximated the values back to the original as the downstream phase ended. After completing the scan, the rat proceeded as indicated by the centroid's postscan ( 0.2 to $2.0 \mathrm{sec}$ from the scan end) displacement of $4.26 \mathrm{~cm}$. Body (centroid) position time series were used for plotting movement trajectories. Rats covered the entire arena during 90 minutes of exploration. Temporal occupancies ( $\log _{10}$ scaled) superimposed with LHS events showed a strong correlation between regions of high occupancy and occurrence of LHS $(r=0.86, p<0.00001)$ [Figs. 1(b) and 1(c)]. The LHS events were distributed throughout the exploratory session [Fig. 1(d)].

LHS dynamics was characterized by computing the noncumulative probability distribution functions (PDFs) of event lifetimes (duration of LHS from the start to the end), interevent intervals (time between consecutive scans), and event magnitudes (sum of absolute instantaneous angular deviations for duration of scan). Figure 1(e) shows the power law distributed probabilities of LHS lifetimes, interevent intervals, and magnitudes from a representative NC rat. LHS event lifetimes, interevent intervals, and magnitudes followed power laws, and their exponents did not alter much with step size variations.

Power law exponents for PDFs of LHS event lifetimes, interevent intervals, and magnitudes for all the rats with step size variations are summarized in Supplemental Material [28] Table I. We have observed signatures of similar scaling for almost all the rats for LHS variables and an example for comparison of an individual rat's power law with the averaged power law exponent for each of the LHS variables is shown in Supplemental Material [28] Fig. 1. Average power law exponents (mean $\pm \mathrm{SD}$ ) for different step sizes for lifetimes ranged between $-1.3 \pm 0.2$ to $-1.5 \pm 0.2$, for interevent intervals between $-0.9 \pm 0.2$ to $-1.2 \pm 0.2$, and for magnitudes from $-1.4 \pm 0.4$ to $-1.5 \pm 0.4$ in the NC rat. Power law equations for lifetimes, interevent intervals, and magnitudes in the $\mathrm{NC}$ rat are summarized as $P(L) \sim L^{-1.4 \pm 0.2}, P(I) \sim I^{-1.0 \pm 0.2}$, and $P(M) \sim M^{-1.5 \pm 0.3}$, where powers represent average of exponents (mean $\pm \mathrm{SD}$ ) of all the rats and all step sizes pooled together (Table I). Power law distribution of occurrence probabilities of lifetimes and interevent intervals suggest strong temporal correlations existing in the system's dynamics while power law distributed event magnitudes reflect a coexisting critical state in the NC rat. Hence this system's dynamics is liable to be explained in light of the SOC.

In order to validate our findings we have analyzed the LHS dynamics in two behavioral states (PA and AMP) where the spatial exploration is altered, as just observation of power law distribution may not necessarily prove that the LHS behavior is an example of criticality, because power law distributions are not uncommon in various natural phenomena. PA induces a state of below normal spatial exploration phase, while AMP induces a hyperactive spatial exploratory phase, thus providing an opportunity to compare the LHS dynamics in various behavioral states. The detailed analysis of LHS dynamics is described 
(a)

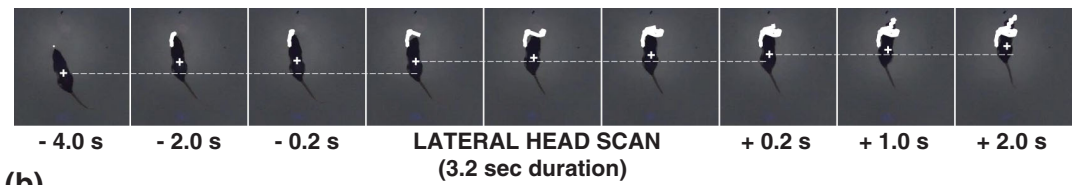

(b)

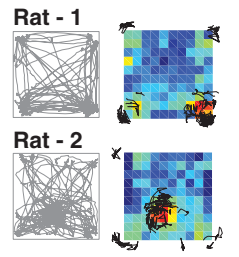

(c)

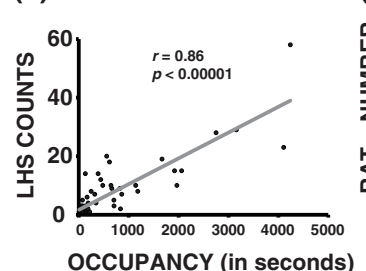

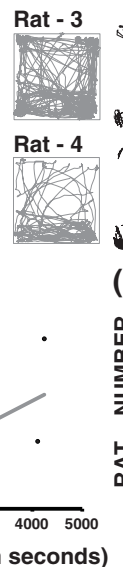

(3.2 sec duration)
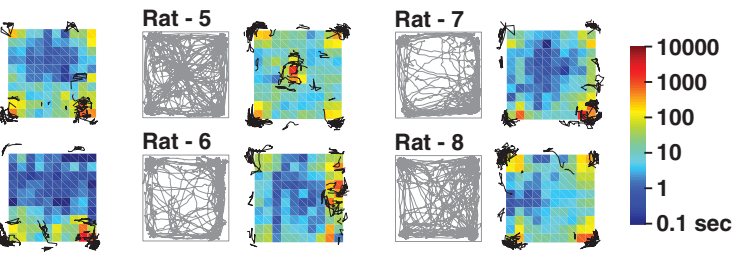

(d)

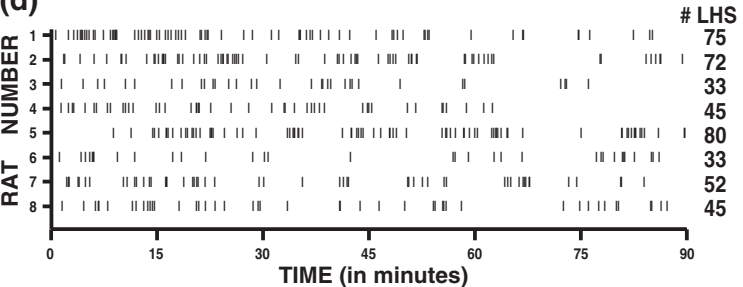

(e) Scale invariant distribution in LHS variables.

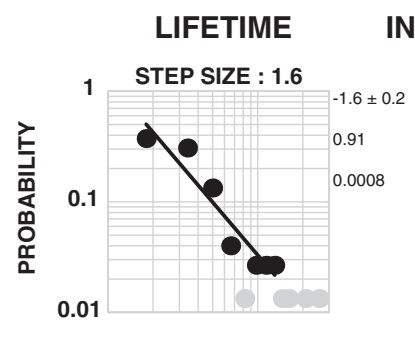

INTER-EVENT INTERVAL
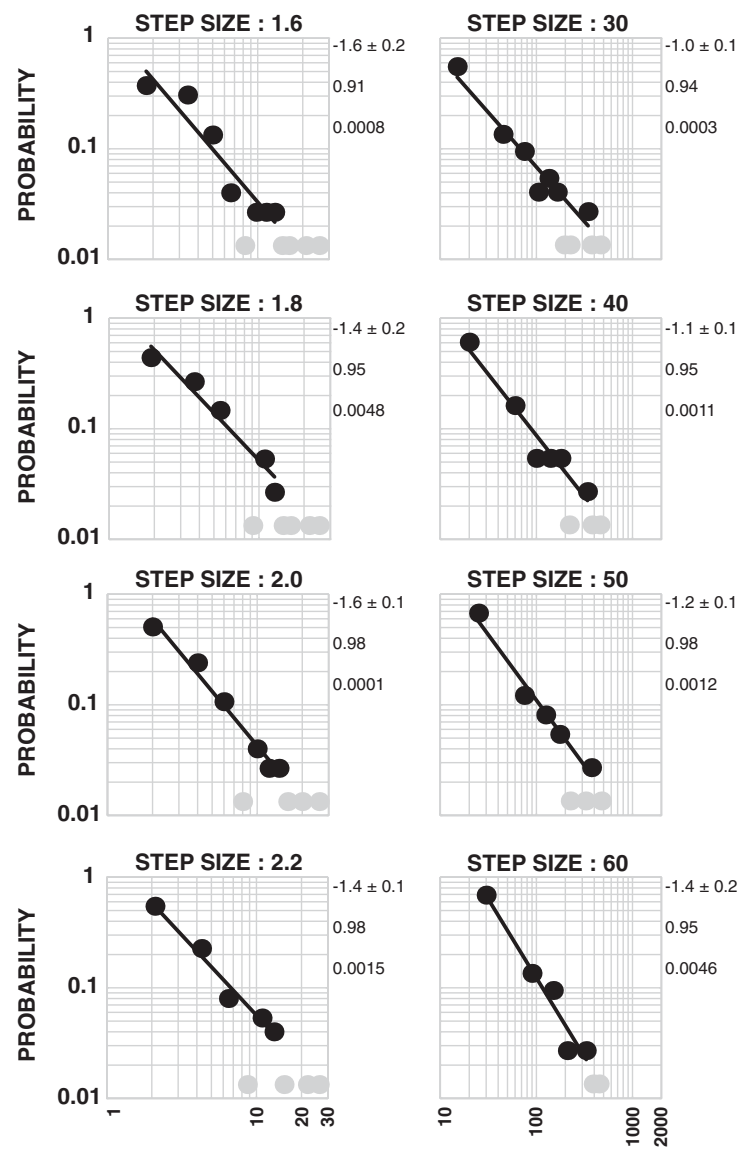

LIFETIME (in sec)

INTER-EVENT INTERVAL (in sec)
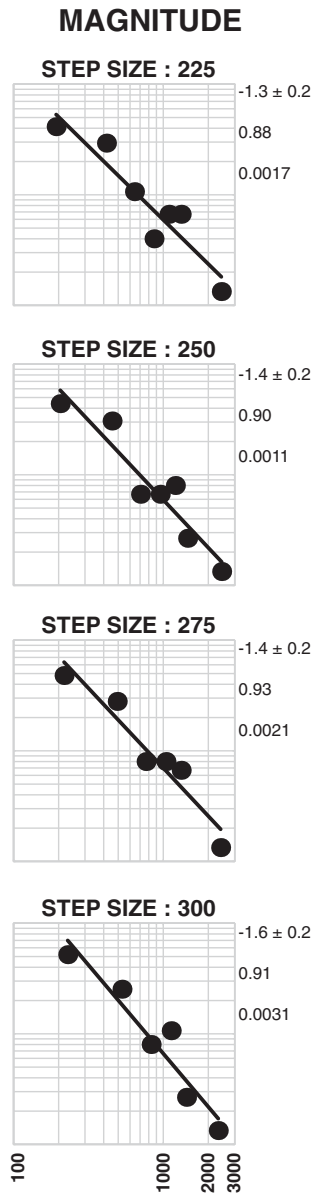

MAGNITUDE $\left(\sum_{s s}^{s e}|\Delta \theta|\right)$
FIG. 1. Spatiotemporal distribution of LHS behavior. [(a)-(d)] LHS profile during open field exploration. (a) A representative LHS during the pause along with its movement before and after the scan. Note the forward shift in the animal's body position $(+)$ before and after the LHS; there is a directional change in nose trajectory (trailing white line) only during the LHS. (b) Movement trajectories (in grey) and temporal occupancy maps (units in seconds, color coded in log scale) of individual rats, superimposed with LHS (in black) during 90 minutes of exploration. (c) Correlation between LHS counts and respective occupancies of all the rats with fit line for linear regression. (d) Distribution of LHS over time, and LHS counts for each rat. (e) Scale invariant distribution in LHS variables. Probability distribution functions of LHS lifetimes, interevent intervals, and magnitudes [sum of absolute instantaneous angular deviations $\left(\sum|\Delta \theta|\right)$ from start $(s s)$ to end ( $s e$ ) of each LHS], shown for a representative rat with four different step sizes, plotted in log-log scale. Black dots represent power law scaled probabilities, while grey dots represent out scaled probabilities. The values next to each panel are the power law exponent \pm standard error, coefficient of determination $\left(R^{2}\right)$ and significance of the exponent ( $p$ value). 
TABLE I. Probability distributions of LHS variables. Comparison of averaged power law exponent (mean \pm SD) for lifetimes, interevent intervals, and magnitudes calculated for different step sizes from all the subjects, and power law equations (average of exponents of all the rats and all the step sizes pooled together) under different behavioral states. LHS variables under PA did not exhibit power laws, and most of the LHS variables under AMP showed signatures of some distinct scale, as compared to NC. Values in bold refers to power law values in NC group.

\begin{tabular}{|c|c|c|c|c|c|}
\hline Lifetime & Step size 1.6 & Step size 1.8 & Step size 2.0 & Step size 2.2 & Power law \\
\hline Normal & $-1.3 \pm 0.2$ & $-1.3 \pm 0.2$ & $-1.5 \pm 0.1$ & $-1.5 \pm 0.1$ & $P(L) \sim L^{-1.4 \pm 0.2}$ \\
\hline Partial Anesthesia & $-1.0 \pm 0.4$ & $-1.2 \pm 0.3$ & $-1.1 \pm 0.4$ & $-1.3 \pm 0.2$ & $P(L) \sim L^{-1.1 \pm 0.3}$ \\
\hline Amphetamine & $-1.7 \pm 0.3$ & $-1.6 \pm 0.2$ & $-1.7 \pm 0.1$ & $-2.1 \pm 0.2$ & $P(L) \sim L^{-1.8 \pm 0.3}$ \\
\hline Interevent interval & Step size 30 & Step size 40 & Step size 50 & Step size 60 & Power law \\
\hline Normal & $-0.9 \pm 0.2$ & $-1.0 \pm 0.1$ & $-1.1 \pm 0.1$ & $-1.2 \pm 0.2$ & $P(I) \sim I^{-1.0 \pm 0.2}$ \\
\hline Partial Anesthesia & $-0.4 \pm 0.3$ & $-0.6 \pm 0.4$ & $-0.7 \pm 0.3$ & $-0.8 \pm 0.4$ & $P(I) \sim I^{-0.6 \pm 0.4}$ \\
\hline Amphetamine & $-1.1 \pm 0.3$ & $-1.2 \pm 0.4$ & $-1.4 \pm 0.4$ & $-1.4 \pm 0.1$ & $P(I) \sim I^{-1.3 \pm 0.3}$ \\
\hline Magnitude & Step Size 225 & Step Size 250 & Step Size 275 & Step Size 300 & Power law \\
\hline Normal & $-1.4 \pm 0.3$ & $-1.4 \pm 0.4$ & $-1.5 \pm 0.3$ & $-1.5 \pm 0.4$ & $P(M) \sim M^{-1.5 \pm 0.3}$ \\
\hline Partial Anesthesia & $-0.7 \pm 0.4$ & $-0.5 \pm 0.4$ & $-0.6 \pm 0.5$ & $-0.7 \pm 0.6$ & $P(M) \sim M^{-0.6 \pm 0.4}$ \\
\hline Amphetamine & $-1.6 \pm 0.2$ & $-1.6 \pm 0.3$ & $-1.6 \pm 0.3$ & $-1.7 \pm 0.3$ & $P(M) \sim M^{-1.6 \pm 0.2}$ \\
\hline
\end{tabular}

in Supplemental Material [28]. In both PA and AMP conditions, rats performed LHS spanning the entire duration of exploration [Supplemental Material [28] Figs. 2(a) and 2(b)], similar to that observed in $\mathrm{NC}$ rats, allowing us to make valid comparison of LHS dynamics across different conditions. On average NC rats had $54.37 \pm 18.85$ LHS $(n=8)$ over a period of 90 minutes of spatial exploration [Fig. 1(d)], while in PA rats we observed $26.75 \pm 8.73$ LHS (49.20\% of NC) and in AMP rats we observed $112 \pm 44.40$ LHS (206\% of NC) (values are mean \pm SD) [Supplemental Material [28] Figs. 2(a) and 2(b)]. The LHS counts (the fundamental basis of LHS dynamics) in NC rats indicate a state of LHS behavior lying between two other behavioral states (PA and AMP conditions), warranting further

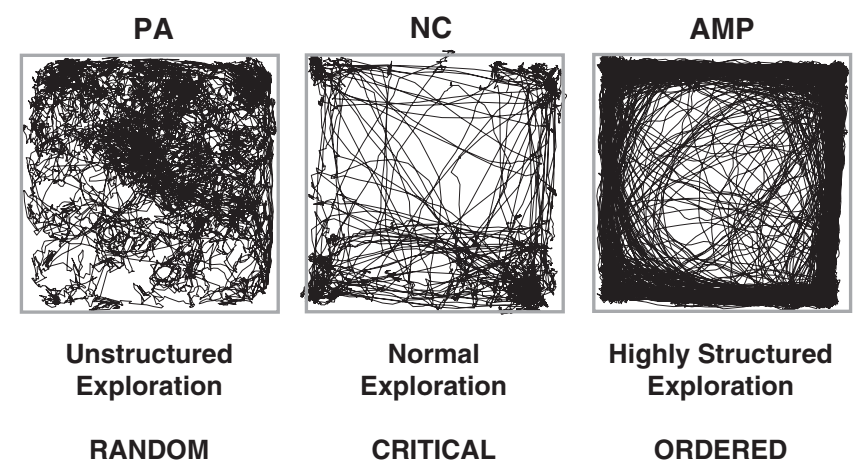

FIG. 2. Comparison of exploratory patterns in different behavioral states. The movement trajectory of an example rat each under different behavioral states (full duration of $90 \mathrm{~min}$ ) revealing transition through the critical phase of exploration under normal conditions, with unstructured (random) or highly structured (ordered) explorations under the influence of partial anesthesia and amphetamine. analysis of LHS variables. Further, PA and AMP rats covered a total distance of $150.87 \pm 47.85$ and $734.27 \pm$ 137.58 meters, respectively, during the exploratory session as compared to $109.86 \pm 62.24$ meters in the case of $\mathrm{NC}$ rats (values are mean $\pm \mathrm{SD}$ ), providing a rate of $0.18 \mathrm{LHS} /$ meter in PA, $0.15 \mathrm{LHS} /$ meter in AMP compared to $0.49 \mathrm{LHS} /$ meter in NC rats, suggesting a distinct role for LHS regarding information processing and decision making during spatial exploration in the normal behavioral state. PA rats showed a relatively unstructured excursions (random) during exploration with random movements under the influence of anesthesia indicating absence of a usual homing behavior [Supplemental Material [28] Fig. 3(a)]. AMP rats covered much longer distances with a higher number of LHS events, moving continuously without staying at a specific location (ordered) for cumulatively longer durations [Supplemental Material [28] Fig. 3(b)], indicating a highly structured behavior unlike NC rats, which exhibit structured excursions with establishment of home base(s) in the normal behavior state [Fig. 1(b)]. The movement trajectory of a rat at each condition is shown in Fig. 2 to compare the exploratory pattern under different behavioral states, clearly indicated the transition through a critical phase.

Noncumulative PDFs for all three LHS variables in PA and AMP rats were computed similar to NC rats for direct comparison. Supplemental Material [28] Fig. 4 shows comparison of probability distributions of LHS lifetimes [Supplemental Material [28] Fig. 4(a)], interevent intervals [Supplemental Material [28] Fig. 4(b)], and magnitudes [Supplemental Material [28] Fig. 4(c)] from representative rats, across different behavioral states. LHS variables under PA did not exhibit power laws, and most of the LHS 
variables under AMP showed signatures of some distinct scale, as compared to the power law distributions observed in NC rats. The slope values for PDFs of LHS variables for all the PA and AMP rats with step size variations are summarized in Supplemental Material [28] Tables II and III, respectively. As summarized in Table I, the exponents for PDFs in the case of PA and AMP clearly deviated from the power law distributions observed in the $\mathrm{NC}$ case (critical phase), suggesting a sweep through two different phases (random and ordered) when the exploratory behavior is tuned to a different state.

Universality, as described through unifying physical principles, has emerged as a connecting link between various natural phenomena. It is an important development in modern science enabling advances in understanding of one system's dynamics to solve complexities in another system. This featured dynamics is attributable to universality through the concept of self-organized criticality having extended applications in physical, biological, and natural systems ranging from magnetic spins to solar flares. Scale invariance in neuronal dynamics reveals information processing mechanisms employed by the brain $[7,8,29]$. It has been shown that specific cortical states (i.e., critical state) maximize the dynamic range of somatosensory information processing [30], and a correlation between behavioral scaling laws and neuronal dynamics [31] has also been reported. Similar mechanisms may be optimizing spatial information management during LHS behavior before it is being presented for downstream processing by specific neural networks involved in spatial navigation. Physiological evidences like potentiation of place fields and increased theta power at LHS sites [27] suggest further the possibility of finding a universal connect between LHS dynamics and a sensory information management system, for efficient processing of spatial information. The information management capacity with a limited number of LHS during exploration leads to immediate assumptions that the system might be behaving in such a way that its information handling capabilities scaled many folds as compared to the system's elementary (LHS here) scales, which can be achieved only if its dynamics are evolved at a critical point in a scale invariant manner.

Our findings of scale invariant LHS behavior, illustrated through power law distributions of LHS probabilities for their magnitudes, durations, and interevent intervals, provide direct evidence for efficient sensory information management via lateral head scanning dynamics during spatial exploration. Power laws reported in our study illustrate long-range temporal correlations suggesting a coexisting critical state (SOC) capable of achieving this, as has been shown in neuronal systems $[6,7]$ and cognitive processes dealing with sensory information [8]. The scaling exponent for LHS magnitudes observed here $(-1.5)$ was similar to that of neuronal systems attributing unit branching parameter maximizing interelement interactions and giving rise to a complex state $[7,32]$. This complex state is a state where elements interact with each other to share information content, and an extension of information handling takes place. Further, this exponent lies within the Barkhausen effect's range of -1 to -2.8 wherein magnetic spins affect the nearby spins at a critical temperature [33]. Similarly, LHS events may interact with each other at a critical point achieved in a completely novel environment, which can be explained either through scaling exponents and high frequency of LHS at regions of higher occupancy observed in our study or through place field potentiation taking place at LHS sites [27]. Do specific locations bind more sensory information that is shared by multiple head scans? If they do, then this could be one of the explanations of how a limited number of scans could manage enormous sensory information. Further, our observations of scale invariance in LHS dynamics are close to the one predicted by SOC in random networks, wherein the "adaptability" contributes to the robustness of the critical state, and the interacting units of such networks coevolve by maintaining an equilibrium [34], facilitating self-organization of networks through gradual evolution [35].

Along with LHS magnitude, scale invariant lifetimes and interevent intervals also suggested this critical point. The exponent for LHS lifetimes was less than -2 , which has been reported for lifetimes of neuronal avalanches also [7]. This means that LHS duration probabilities decay slower than the neuronal avalanches; hence, it appears to be a robust system. LHS events of smaller duration were more frequent than long lasting events. This was another confirmation of LHS dynamics being a critical system. Though many systems like earthquakes have been shown to exhibit unified scaling for recurrence times between large events [36], we have observed power law distribution for LHS interevent intervals with an exponent near -1 , computed without any event threshold, behaving similar to Omori sequences observed for small events in seismic processes [37], and for smaller waiting times in neuronal spike avalanches [38]. However, this exponent was different from the power law exponent for recurrence time distribution (approximately -2 ) computed for the speed profile of freely moving rats [39]. This may be due to a difference in methods used for computing PDFs. We have computed PDFs for interevent intervals without applying any event size threshold but the same were applied for computing PDFs for waiting times between large events in other cases. In our case, each and every event had its own importance and could influence the temporal structure of LHS occurrence; hence, we analyzed interevent intervals instead of waiting or recurrence times. However, it is to be noted that the waiting times PDF was Poisson distributed when Bak Tang and Wiesenfeld (BTW)'s original SOC model was used [40], while Sánchez et al. [41] have rejected power law distribution of waiting times as an essential feature of SOC systems. But at the minimal note on interevent intervals, it is evident that they exhibit long-range temporal correlations required to hold the system in a precise state. 
Since the pharmacological interventions in both PA and AMP conditions affected spatial exploration and associated LHS dynamics, we were able to identify distinct phases on either side of critical LHS dynamics in normal condition. In case of partial anesthesia, where the animals' senses were diminished, the scale free properties of LHS behavior were compromised, whereas amphetamine treated rats exhibited a hyperactive state with occurrence of more LHS events and revealing signatures of some distinct scale above normal power law exponents. In order to achieve a critical state the system has to be balanced somewhere between an inhibited and an excited state. The exploratory architecture under the normal behavior state was neither random (as in the case of PA) nor too ordered (as in the case of AMP), supporting active decision making in NC rats reflected in their LHS dynamics. Our observations in rats with partial anesthesia or amphetamine indicate subcritical or supercritical phases in LHS dynamics diverging from a transit phase of criticality, thus providing additional evidence to suggest that the LHS behavior is a scale free phenomenon, exhibiting features of criticality having functional significance.

We conclude that these distributions collectively provide evidence for a critical point in optimizing the sensory information by lateral head scanning behavior in order to facilitate its foolproof processing down the stream by adopting scale invariant universal mechanisms, suggesting a connect between criticality, information processing, and spatial cognition in animals under normal conditions.

This work was supported by Institutional Core funding from National Brain Research Centre, India. We would like to acknowledge Dr. Joseph D. Monaco, Johns Hopkins School of Medicine, Baltimore, USA and Dr. Collins Assisi, IISER-Pune, India, for the scientific discussions. We are thankful to Professor K. P. Mohanakumar, Director, IUCBR\&SSH, Kottayam, India, for the generous gift of amphetamine.

*Corresponding author. yoga@nbrc.ac.in

[1] M. R. Schroeder, Fractals, Chaos, Power Laws: Minutes from an Infinite Paradise (Courier Corporation, 2012).

[2] P. Bak, C. Tang, and K. Wiesenfeld, Phys. Rev. Lett. 59, 381 (1987).

[3] P. Bak, C. Tang, and K. Wiesenfeld, Phys. Rev. A 38, 364 (1988).

[4] P. Bak, How Nature Works: The Science of Self-Organized Criticality(Copernicus, New York, 1999).

[5] T. Gisiger, Biol. Rev. Camb. Philos. Soc. 76, 161 (2001).

[6] J. M. Beggs, Phil. Trans. R. Soc. A 366, 329 (2008).

[7] J. M. Beggs and D. Plenz, J. Neurosci. 23, 11167 (2003).

[8] O. Kinouchi and M. Copelli, Nat. Phys. 2, 348 (2006).

[9] A. Proekt, J. R. Banavar, A. Maritan, and D. W. Pfaff, Proc. Natl. Acad. Sci. U.S.A. 109, 10564 (2012).
[10] D. Eilam, Neurosci. Biobehav. Rev. 47, 393 (2014).

[11] E. C. Tolman, Psychol. Rev. 55, 189 (1948).

[12] D. Eilam and I. Golani, Behav. Brain Res. 34, 199 (1989).

[13] O. Tchernichovski and I. Golani, J. Neurosci. Methods 62, 21 (1995).

[14] D. G. Wallace, D. J. Hines, and I. Q. Whishaw, J. Neurosci. Methods 113, 131 (2002).

[15] C. R. Gallistel, The Organization of Learning (A Bradford Book, Cambridge, 1993).

[16] J. S. Taube, Prog. Neurobiol. 55, 225 (1998).

[17] M. A. A. Van der Meer, Z. Richmond, R. M. Braga, E. R. Wood, and P. A. Dudchenko, Behav. Neurosci. 124, 164 (2010).

[18] J. J. Knierim, H. S. Kudrimoti, and B. L. McNaughton, J. Neurophysiol. 80, 425 (1998).

[19] D. G. Wallace, M. M. Martin, and S. S. Winter, Naturwissenschaften 95, 1011 (2008).

[20] D. Drai, N. Kafkafi, Y. Benjamini, G. Elmer, and I. Golani, Behav. Brain Res. 125, 133 (2001).

[21] C. H. Vanderwolf, Electroencephalogr. Clin. Neurophysiol. 26, 407 (1969).

[22] D. Eilam, M. Dank, and R. Maurer, Behav. Brain Res. 141, 73 (2003).

[23] I. Golani, Y. Benjamini, and D. Eilam, Behav. Brain Res. 53, 21 (1993).

[24] D. L. Kramer and R. L. McLaughlin, American Zoologist 41, 137 (2001).

[25] F. Nemati and I. Q. Whishaw, Behav. Brain Res. 182, 119 (2007).

[26] I. Q. Whishaw, J. C. Cassel, M. Majchrzak, S. Cassel, and B. Will, Hippocampus 4, 577 (1994).

[27] J. D. Monaco, G. Rao, E. D. Roth, and J. J. Knierim, Nat. Neurosci. 17, 725 (2014).

[28] See Supplemental Material at http://link.aps.org/ supplemental/10.1103/PhysRevLett.118.158104, for description of experimental methods, data acquisition and analysis; figures and tables; and additional data under PA and AMP conditions.

[29] R. Legenstein and W. Maass, Neural Netw. 20, 323 (2007).

[30] S. H. Gautam, T. T. Hoang, K. McClanahan, S. K. Grady, and W. L. Shew, PLoS Comput. Biol. 11, e1004576 (2015).

[31] J. M. Palva, A. Zhigalov, J. Hirvonen, O. Korhonen, K. Linkenkaer-Hansen, and S. Palva, Proc. Natl. Acad. Sci. U.S.A. 110, 3585 (2013).

[32] J. M. Beggs and N. Timme, Front. Physiol. 3, 163 (2012).

[33] H. Barkhausen, Z. Phys. 20, 401 (1919).

[34] P. Bak and K. Sneppen. Phys. Rev. Lett. 71, 4083 (1993).

[35] K. Christensen, R. Donangelo, B. Koiller, and K. Sneppen. Phys. Rev. Lett. 81, 2380 (1998).

[36] Á. Corral, Phys. Rev. Lett. 92, 108501 (2004).

[37] F. Omori, J. Coll. Sci. Imp. Univ. Tokyo 7, 111 (1894).

[38] T. L. Ribeiro, M. Copelli, F. Caixeta, H. Belchior, D. R. Chialvo, M. A. L. Nicolelis, and S. Ribeiro, PLoS One 5, e14129 (2010).

[39] C. K. Yadav, M. K. Verma, and S. Ghosh, BioSystems 102, 77 (2010).

[40] G. Boffetta, V. Carbone, P. Giuliani, P. Veltri, and A. Vulpiani, Phys. Rev. Lett. 83, 4662 (1999).

[41] R. Sánchez, D. E. Newman, and B. A. Carreras, Phys. Rev. Lett. 88, 068302 (2002). 